\title{
INNULs: A Novel Design Amplification Strategy for Retrotransposable Elements for Studying Population Variation
}

\author{
Bobby L. LaRue $^{a}$ Sudhir K. Sinhab Anne H. Montgomery ${ }^{b}$ Robyn Thompson ${ }^{b}$

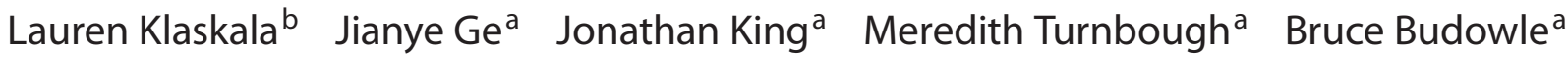 \\ a Institute of Applied Genetics, Department of Forensic and Investigative Genetics, University of North Texas \\ Health Science Center, Fort Worth, Tex., and ${ }^{\mathrm{b}}$ InnoGenomics Technologies, LLC, New Orleans, La., USA
}

\section{Key Words}

INDELs $\cdot$ INNULs $\cdot$ SNPs $\cdot$ Alu $\cdot$ Long interspersed nuclear elements $\cdot$ Genotyping

\begin{abstract}
Objectives: Retrotransposable elements (REs), consisting of long interspersed nuclear elements (LINEs) and short interspersed nuclear elements (SINEs), are a group of markers that can be useful for human identity testing. Until now, however, due to the inherent size difference (up to $6 \mathrm{~kb}$ in some instances) associated with insertion and null alleles (or INNULs), the use of REs for facilitated population studies has not been sought or practical. The size of the insertion elements (from a few hundred to several thousand bp) has proven to limit their utility as a marker because of the inefficient amplicon yield with PCR. A novel primer design now facilitates INNUL marker testing. A preliminary panel of single-locus markers was developed to evaluate the potential of typing these insertion elements. Nine INNULs (5 Alu and 4 LINEs) were typed in three major North American populations and analyzed for population genetic features. In addition, the variation of each marker among the sample populations provides insight of its potential use as individual identification or ancestral marker. Methods: INNUL markers were
\end{abstract}

developed into fluorescently labeled single-loci PCR. Nine markers were developed with amplicons that were less than $180 \mathrm{bp}$ in length, and, depending on the locus amplicons of the INNULs, alleles varied in size from 50 to $1 \mathrm{bp}$. This allele size is noteworthy because the insertion alleles of the 9 loci range in size from 297 to 6,195 bp. The allele distribution of the INNULs was assessed and analyzed in three major North American populations. Results: Upon observation of the distribution of the alleles in three major North American populations, the markers generally met Hardy-Weinberg expectations, and there was little evidence of detectable levels of linkage disequilibrium. Due to varying distributions of the alleles in the major population groups tested, some of the markers might be better suited for use as an individual identification marker, while others are better suited for bio-ancestral studies. Conclusions: Using the primer design strategy described in our work, SINEs and (for the first time, to our knowledge) LINEs can be utilized as markers for studying population genetic variation that is more amenable to the limitations of the PCR technique. This study lays the foundation for future work of developing a multiplex panel of INNUL markers that can be used as a single-tube assay for human identity testing utilizing small amplicons $\quad<180$ bp), which could be useful for ancient or degraded forensic DNA samples.

Copyright ๑ 2012 S. Karger AG, Basel

\section{KARGER}

Fax +4161306 1234 E-Mail karger@karger.ch www.karger.com

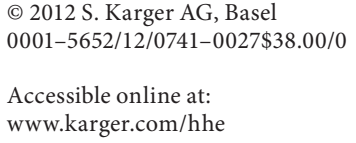

Bobby LaRue

Institute of Applied Genetics

University of North Texas Health Science Center

Fort Worth, TX 76107 (USA)

E-Mail bobby.larue@unthsc.edu 


\section{Introduction}

Short tandem repeat (STR) loci are the primary genetic markers used in human identity testing. These markers are highly polymorphic and afford a high degree of sensitivity of detection such that relatively low quantities (from $1 \mathrm{ng}$ to $250 \mathrm{pg}$ ) of template DNA can be analyzed [1-6]. Retrotransposable elements (REs) consisting of long interspersed nuclear elements (LINEs) and short interspersed nuclear elements (SINEs) are another group of markers that could be useful for population genetic and evolutionary studies, as well as human identity testing [7-13]. Ustyugova et al. [14] demonstrated that REs could be used for cell line identification. Novick et al. [15] and Mamedov et al. [16] recently described a set of Alus (a type of SINE) for paternity testing. Both of these studies intimated that the systems could be applied to forensic analyses. REs have low mutation rates, which make them appealing for population studies and missing persons kinship analyses compared with the less stable STRs. Although REs make up over $40 \%$ of the human genome [17] and present myriad possible targets for population studies and potentially human identity testing, these insertion elements have received limited attention for use in human identity testing. We have termed the Alus and LINEs as INNULs instead of INDELs as the two allelic states are known to be an insertion and null. Indeed, some INDELs are similar in this regard but may be more difficult to describe in this manner. Thus, INNUL better describes the already well-established allelic states of these markers.

Obtaining a comparable yield of products for both alleles of a heterozygote during PCR typically requires similar amplicon sizes. The difference in size of a null allele and an insertion element generally is quite large from the perspective of PCR efficiency. The preferential amplification of the smaller allele often makes PCR typing of such markers problematic. In addition, some materials such as forensic samples often are compromised in quality and quantity. Degraded samples may contain fragments of DNA that are less than the length of the insertion element [18-21].

REs can range in size from hundreds of (SINEs) to several thousand (LINEs) base pairs (bp) in length [7-13]. Previous attempts to use Alu sequences for identity testing capitalized on the size difference between insertion and null alleles by amplifying the entire region with the same forward and reverse primers [15]. The insertion allele would be $200-400$ bp larger than the null allele and could be detected electrophoretically based on size differ- ences. While useful for paternity testing and some population studies where DNA quality is not compromised, the large size difference between amplicons of the null and insertion alleles does impact amplification efficiency during the PCR technique, which is a limitation for the design of efficient genetic typing assays (i.e., multiplexes) and for forensic sample analyses. Thus, the use of REs has not been embraced for routine application diagnostics [22].

A novel primer design described herein and referred to subsequently as Mini-Primer strategy reduces the overall amplicon size as well as the difference in amplicon sizes between the two allelic states of INNULs. Amplification of the two alleles occurs through a common unlabeled forward primer and two fluorescently labeled reverse primers. The labeled reverse primer for the null allele overlaps the insertion site of the RE, and the labeled reverse primer for the insertion allele has an overlap region with the junction and the RE itself, or just inside the RE (fig. 1). With this design, the resulting INNUL allelic amplicons can be designed to differ by as little as $1 \mathrm{bp}$, and because each allelic-state reverse primer is labeled with a different fluor, the amplicons can even be the same size. Additionally, the amplicon size can be reduced substantially, to a size much smaller than currently used STR markers, such that substantially degraded samples can be typed. With this design, a more simplified and automated technology can be applied for LINE and SINE typing.

Selection criteria for INNUL markers depend on the application. Markers that are highly polymorphic in all major populations (i.e., approaching $50 \%$ heterozygosity) are desirable for human identity testing [4, 6, 23-25], while those demonstrating high coefficients of inbreeding (e.g., SNPs in which the different allelic states approach fixation in different populations) can be used for bio-ancestral analyses [26]. To demonstrate the potential of the newly designed primer sets for population studies and potentially for human identity testing, an initial set of INNUL markers based on Alus and LINEs was chosen. The Alu-based INNUL markers were selected based on molecular characteristics and extant population data [27-29]. There were no available population data on the LINE INNUL markers, so only molecular characteristics were used as selection criteria for this study. The markers' amplicons range in size between approximately 50 and $179 \mathrm{bp}$, and individual INNULs differ in amplicon size between 1 and $54 \mathrm{bp}$. Population studies were performed to assess diversity within and among major population groups to determine the applicability of the selected INNULs for either identity testing or bio-ancestral analyses. 


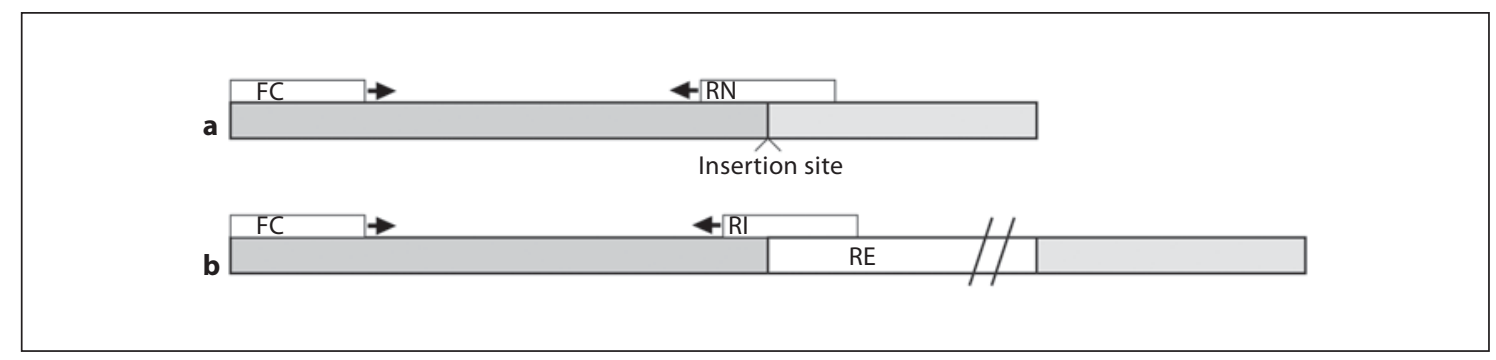

Fig. 1. Amplification strategy for INNUL typing. a A common forward primer (FC) is used for both insertion and null alleles. A fluorescently labeled 'null-specific' reverse primer (RN) straddles the insertion site of the RE and anneals in the absence of the RE. $\mathbf{b}$ In instances where the RE is present, the annealing site of the reverse primer is disrupted, and the 'insertion-specific' reverse primer (RI) anneals at the site that overlaps with the insertion site and the adjacent portion of the RE.

\section{Materials and Methods}

\section{Marker Selection}

Markers were selected from dbRIP, existing literature, and through BLAST sequence analysis [7-16, 27, 30]. After the initial selection, the potential loci were assessed for their suitability for the primer design [22].

\section{Primer Design and Preparation}

Primers were designed using Primer3 (input version 0.4.0. http://frodo.wi.mit.edu/primer3/). A set of three primers was designed for each marker: one forward primer and two reverse primers, one for the insertion and one for the null allele. All designed primers have $\mathrm{T}_{\mathrm{m}}$ values in the range of $58-61^{\circ} \mathrm{C}$. The program Reverse Complement from the Harvard Medical Technology Group and Lipper Center for Computational Genomics (arep. med.harvard.edu/) was used. Subsequently, the primers were screened against the GenBank non-redundant database to determine if they were unique DNA sequences.

The fluorescently labeled and unlabeled oligonucleotide primers were synthesized either by Bio-Synthesis, Inc. (Dallas, Tex., USA) or by Integrated DNA Technologies, Inc. (Coralville, Iowa, USA). All lyophilized primers were dissolved in $10 \mathrm{mM}$ TE (Tris and EDTA) buffer ( $\mathrm{pH} 8.0$ ) to a $100 \mu \mathrm{M}$ stock concentration $(10 \times)$. The stock primers were stored at $4^{\circ} \mathrm{C}$ until used. Following reconstitution, each primer was diluted using TE buffer to a final concentration of $10 \mu \mathrm{M}(1 \times)$. Each primer mix consisted of three primers: one unlabeled forward primer and two labeled reverse primers, with twice the amount of the forward primer than the reverse primers $(1.0,0.5$, and $0.5 \mu \mathrm{l}$, respectively). All labeled primers were stored in opaque polypropylene tubes to avoid quenching of the fluorescent tags.

\section{Data Analysis for ABI 310}

After amplification, samples were prepared by combining 20 $\mu \mathrm{l}$ of $\mathrm{Hi}-\mathrm{Di}^{\mathrm{TM}}$ formamide, $0.25 \mu \mathrm{l}$ of ROX 350 size standard, and $1 \mu \mathrm{l}$ of DNA product per reaction. Samples were incubated at $95^{\circ} \mathrm{C}$ in a water bath for $3 \mathrm{~min}$. Separation and detection of STR amplification products were performed on an ABI Prism ${ }^{\circledR} 310$ Genetic Analyzer (Life Technologies) using the following parameters for the GS STR POP4 $(1 \mathrm{ml})$ F module: injection at $15 \mathrm{kV}$ for
$5 \mathrm{~s}$, separation at $60^{\circ} \mathrm{C}$, run time of $24 \mathrm{~min}$. Data were analyzed using the GeneMapper ID-X Software version 3.2 (Life Technologies).

\section{Population Samples}

Buccal samples from 279 African-Americans, Caucasians, and southwestern Hispanics were either collected from unrelated individuals residing in Texas or were kindly provided by Genetic Testing Laboratories (Las Cruces, N. Mex., USA). The samples were anonymized and collected in accordance with methods approved by the Institutional Review Board of the University of North Texas Health Science Center in Fort Worth, Tex., USA. Population affinity was determined by self-declaration.

\section{DNA Extraction}

DNA was isolated from buccal swabs using either the AutoMate Express ${ }^{\circledR}$ (Life Technologies) or the DNA Investigator ${ }^{\circledR}$ Kit (Qiagen, Hilden, Germany) according to the manufacturers' recommendations. The quantity of DNA was determined by qPCR using the Quantifiler ${ }^{\circledR}$ Quantification Kit and 7500 Real-Time PCR $^{\circledR}$ System (Life Technologies). Samples were then normalized to $250 \mathrm{pg} / \mu \mathrm{l}$ and stored at either $-20^{\circ} \mathrm{C}$ or $-40^{\circ} \mathrm{C}$ until amplification.

\section{PCR and Electrophoretic Conditions}

For PCR, each locus was amplified as a singleplex assay. A reaction was composed of $12.5 \mu \mathrm{l}$ of a $2 \times$ Amplitaq Gold PCR Master Mix ${ }^{\circledR}$ (Life Technologies): $2.0 \mu$ l of the primer mix (for specifications of primers, see table 1) and $500 \mathrm{pg}$ of template DNA were brought to a final volume of $25 \mu \mathrm{l}$. The resulting reaction mixture was amplified using a Geneamp 9700 PCR System ${ }^{\circledR}$ (Life Technologies) thermocycler. The amplification protocol was an initial step of $94^{\circ} \mathrm{C}$ for $10 \mathrm{~min}$ followed by 32 cycles of $94^{\circ} \mathrm{C}$ for $1.5 \mathrm{~min}$, $60^{\circ} \mathrm{C}$ for $1 \mathrm{~min}$, and $72^{\circ} \mathrm{C}$ for $3 \mathrm{~min}$ followed by an extension step of $72^{\circ} \mathrm{C}$ for $10 \mathrm{~min}$. An additional extension step at $60^{\circ} \mathrm{C}$ for 60 min was added to promote terminal adenylation.

For capillary electrophoresis, $1 \mu \mathrm{l}$ of the amplified product was added to $8.5 \mu \mathrm{l}$ of formamide and $0.5 \mu \mathrm{l}$ of GS ROX 500 ILS $^{\circledR}$ (Life Technologies), mixed, and snap cooled. Capillary electrophoresis was performed on either a 3500xl or 3130xl Genetic Analyzer $^{\circledR}$ (Life Technologies) capillary electrophoresis instrument. 


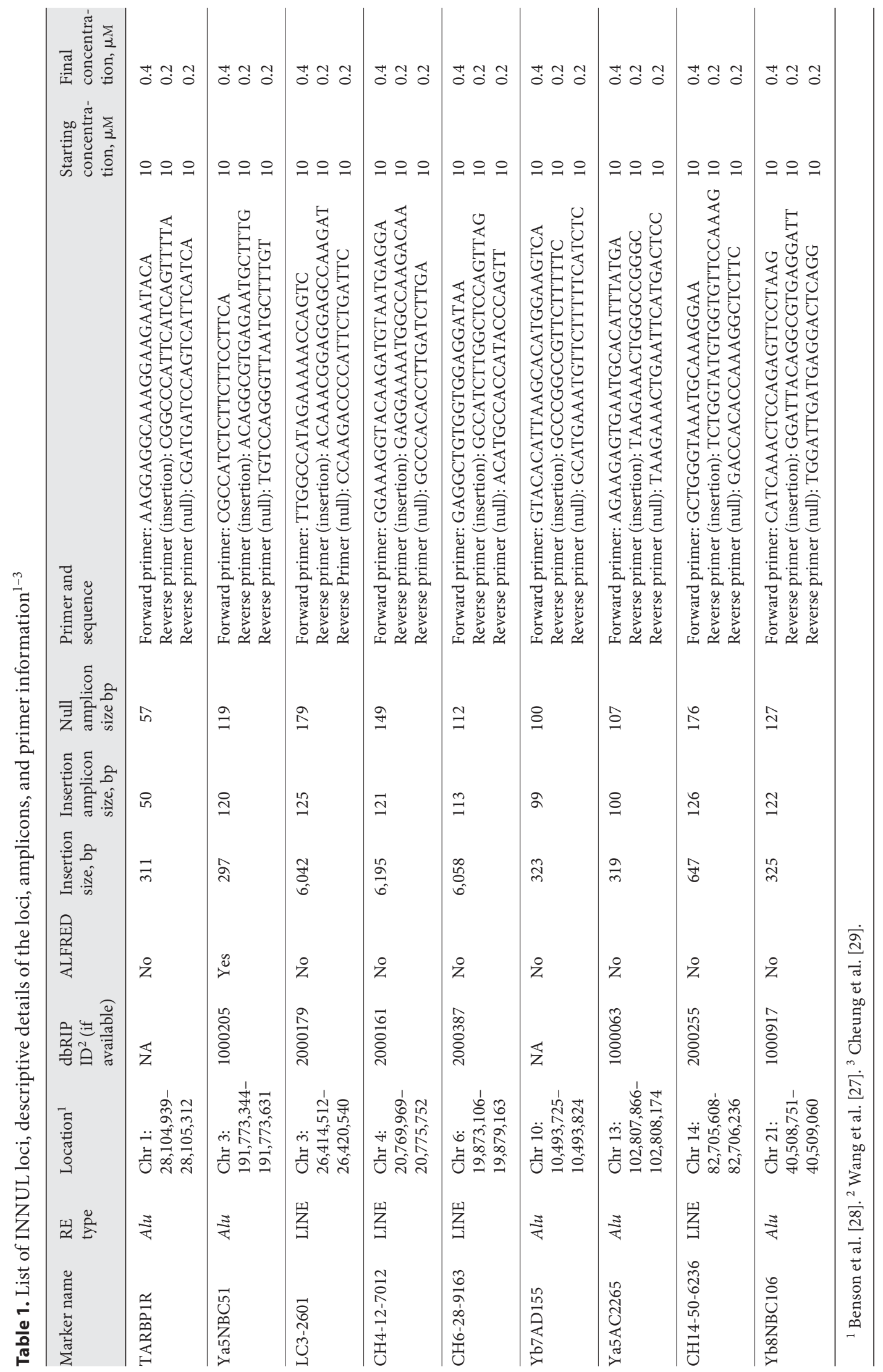


Samples were injected for $24 \mathrm{~s}$ and subjected to electrophoresis at $15 \mathrm{kV}$ in POP4 ${ }^{\mathrm{TM}}$ (Life Technologies). Electrophoretic data were analyzed using GeneMapper IDX ${ }^{\circledR}$ software (Life Technologies).

Population and Statistical Analyses

Population and statistical analyses, including $\mathrm{F}_{\mathrm{ST}}$, were performed with either GDA software [31], Arlequin 3.11 [32], or inhouse developed software. Departures from Hardy-Weinberg equilibrium (HWE) and linkage equilibrium were tested using Fisher's exact test. Bonferroni's correction for multiple comparisons was performed according to Weir and Cockerham [33].

\section{Results and Discussion}

\section{Marker Selection and PCR Optimization}

Initial efforts towards marker selection focused on the set of forensic candidate markers described by Mamedov et al. [16]. Using these markers as a benchmark and the previously described Mini-Primer strategy, an attempt was made to reduce the amplicon size of a subset of markers reported by Mamedov et al. [16]. Primers for 5 markers were designed such that all amplicons were less than $120 \mathrm{bp}$ in size for both the insertion and null alleles (online suppl. table 1; for all online suppl. material, see www. karger.com/doi/10.1159/000343050). Gel electrophoresis was used to visualize the products of the reactions (online suppl. fig. 1). Examples with both insertion and null alleles that differ in size by 7 bp are shown. This result supported the potential of the Mini-Primer strategy. It should be noted that, in this study, each allele was labeled with a different fluor. A long-term goal (beyond the proof-ofconcept study herein) would be to label alleles of a locus with the same fluor to facilitate multiplexing and allele balance. However, at this early stage, it was desirable to have an indicator that could designate each allele to evaluate if estimated sizes were indeed those that were observed, and when alleles were similar in size, they could be discerned properly.

Following this initial success, both Alus and LINES were chosen from the literature $[9,10,14-16,27,30]$. Through trial and error, a set of candidate markers was selected to demonstrate the feasibility of the Mini-Primer approach for typing INNULs. These loci are described in table 1.

The selected markers are 5 Alus and 4 LINEs with amplicons that are between 50 and $179 \mathrm{bp}$ in length. Figure $2 \mathrm{a}, \mathrm{b}$ shows example electropherograms of the size range of alleles for two loci (LC3-2601 and CH6-28-9163, respectively) which range from approximately 50 to $1 \mathrm{bp}$ for the candidate set. An electropherogram for a heterozygous individual at the locus with the largest amplicon is shown in figure $2 \mathrm{a}(179 \mathrm{bp})$ and for a heterozygous individual at the locus with the smallest amplicon (TARBP1R; $50 \mathrm{bp}$ ) in figure 2c. The insertions and null amplicons were in the position and relative size that were predicted. Thus, it is feasible to generate amplified products of the allelic states of Alus and LINEs that are more suited for the limitations of the PCR technique. When the size is similar for amplified products of allelic states, assays tend to be more robust and demonstrate less preferential amplification of the smaller-sized allele.

\section{Population Studies}

Three North American sample populations (AfricanAmerican, $n=93$; Caucasian, $n=93$; southwestern Hispanic, $n=93$ ) were typed for the 9 INNUL loci. The frequencies of the null allele per locus, observed heterozygosity, random match probability, and power of discrimination are listed in table 2 . To the best of our knowledge, these are the first population data reported on LINEs, and, with the exception of the TARPB1R and Ya5NBC51 loci, these are the first population data generated for the Alu markers reported herein. Heterozygosity for the markers ranged from 0.02 to 0.48 in southwestern Hispanics, from 0.03 to 0.59 for Caucasians, and from 0.31 to 0.50 in African-Americans. There was only one departure from HWE detected (at locus CH14-50-6236 in the African-American population). This observed departure was no more than would be expected by chance. When the critical value was adjusted by the Bonferroni correction [33], the departure was no longer significant (table 2).

Departures from linkage equilibrium [i.e., linkage disequilibrium (LD) between pairs of loci] were tested for each of the three populations (online suppl. table 2). Four pairs of loci in the African-American population and one pair in the Caucasian population demonstrated significant LD (at $\mathrm{p}<0.05$ as determined by Fisher's exact test). All of the loci pairs in the African-American population involved locus CH14-50-6236, which was the only locus to demonstrate a detectable departure from HWE (at the 0.05 level). These apparent LDs thus could be associated with the deviation from HWE as described by Falush et al. [34] and Chakraborty [35] or due to substructure in the African-American population. Regardless, when the critical value was adjusted for multiple comparisons (via the Bonferroni correction), none of the loci pairs displayed significant LD.

Wright's $\mathrm{F}_{\mathrm{ST}}$ was estimated to assess the population substructure [33]. For the three populations, the combined $\mathrm{F}_{\mathrm{ST}}$ was 0.122 (table 2). Three loci (CH14-50-6236, 


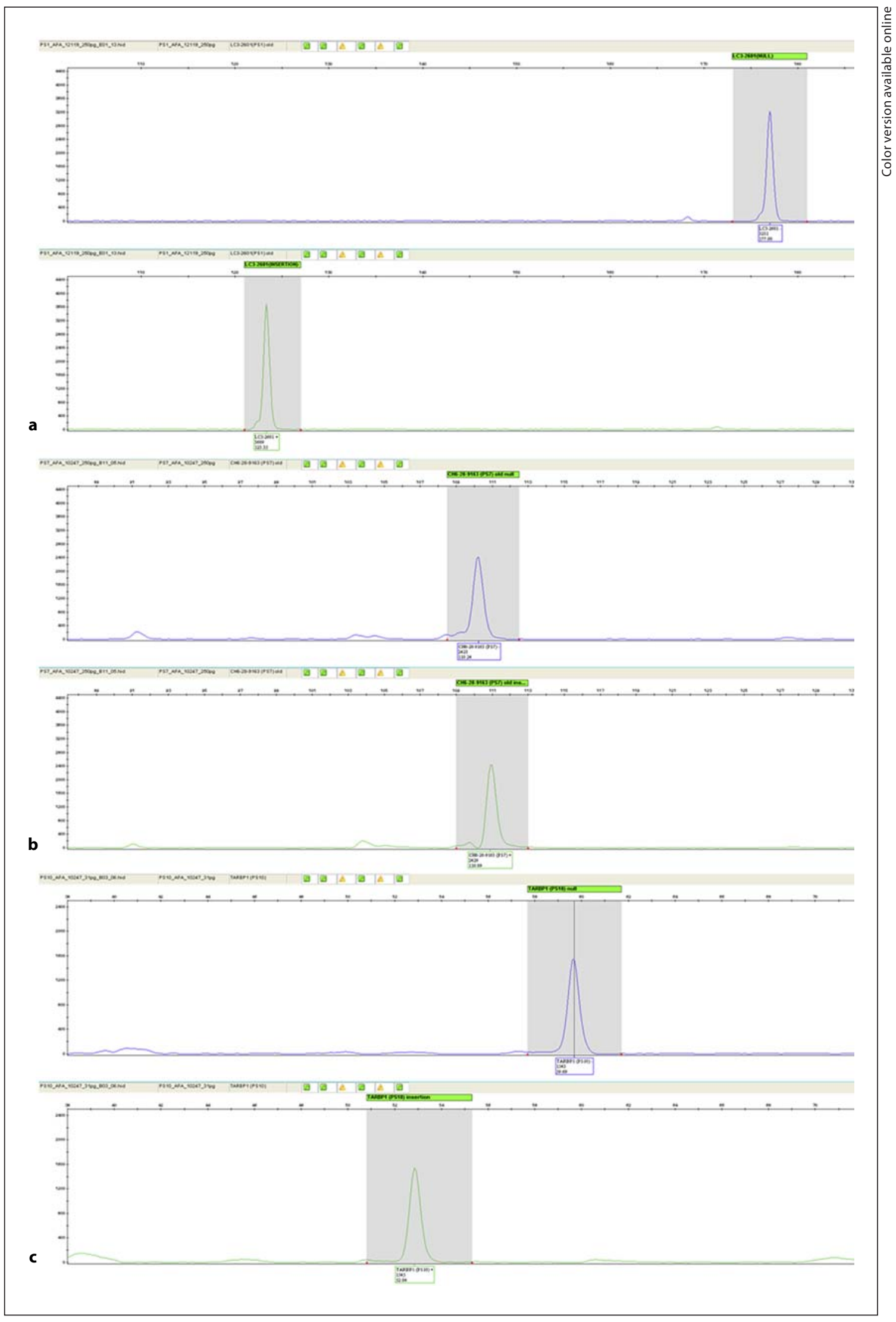


Table 2. Population-specific INNUL allele frequencies, other population parameters, and $\mathrm{F}_{\mathrm{ST}}$ values for alleles across three North American populations $(n=93$ each)

\begin{tabular}{|c|c|c|c|c|c|c|}
\hline Marker & $\begin{array}{l}\text { Frequency } \\
\text { of null }\end{array}$ & $\mathrm{H}_{\mathrm{o}}$ & $\begin{array}{l}\text { HWE } \\
\text { (p value) })^{1,2}\end{array}$ & RMP & PD & $\mathrm{F}_{\mathrm{ST}}^{3}$ \\
\hline \multicolumn{7}{|c|}{ African-Americans } \\
\hline LC3-2601 & 0.5227 & 0.4545 & 0.3884 & 0.3564 & 0.6436 & 0.0560 \\
\hline Yac52265 & 0.7198 & 0.3407 & 0.2006 & 0.4300 & 0.5700 & 0.0715 \\
\hline CH14-50-6236 & 0.4881 & 0.3571 & $0.0094^{*}$ & 0.3345 & 0.6655 & 0.1766 \\
\hline CH4-12-7012 & 0.1978 & 0.3077 & 0.7372 & 0.5170 & 0.4830 & 0.4831 \\
\hline Ya5NBC51 & 0.4205 & 0.4318 & 0.2722 & 0.3605 & 0.6395 & 0.0680 \\
\hline Yb7AD155 & 0.5872 & 0.5000 & 0.8219 & 0.3902 & 0.6098 & -0.0030 \\
\hline CH6-28-9163 & 0.7582 & 0.3956 & 0.5825 & 0.4725 & 0.5275 & 0.2553 \\
\hline Yb8NBC106 & 0.4494 & 0.4944 & 1.0000 & 0.3774 & 0.6226 & 0.0368 \\
\hline TARBP1R & 0.6833 & 0.3889 & 0.3441 & 0.4052 & 0.5948 & 0.0144 \\
\hline Overall & & & & 0.0003 & 0.9997 & 0.1224 \\
\hline \multicolumn{7}{|l|}{ Caucasians } \\
\hline LC3-2601 & 0.0163 & 0.0326 & 1.0000 & 0.9369 & 0.0631 & 0.0560 \\
\hline Yac52265 & 0.2473 & 0.4066 & 0.5600 & 0.4691 & 0.5309 & 0.0715 \\
\hline CH14-50-6236 & 0.7258 & 0.3978 & 1.0000 & 0.4416 & 0.5584 & 0.1766 \\
\hline CH4-12-7012 & 0.0215 & 0.0430 & 1.0000 & 0.9177 & 0.0823 & 0.4831 \\
\hline Ya5NBC51 & 0.4674 & 0.4130 & 0.0978 & 0.3450 & 0.6550 & 0.0680 \\
\hline Yb7AD155 & 0.5440 & 0.5385 & 0.5338 & 0.4003 & 0.5997 & -0.0030 \\
\hline CH6-28-9163 & 0.4674 & 0.5217 & 0.8413 & 0.3887 & 0.6113 & 0.2553 \\
\hline Yb8NBC106 & 0.5000 & 0.5914 & 0.1013 & 0.4332 & 0.5668 & 0.0368 \\
\hline TARBP1R & 0.4355 & 0.5269 & 0.5375 & 0.3978 & 0.6022 & 0.0144 \\
\hline Overall & & & & 0.0016 & 0.9984 & 0.1224 \\
\hline \multicolumn{7}{|c|}{ Southwestern Hispanics } \\
\hline LC3-2601 & 0.0110 & 0.0220 & 1.0000 & 0.9570 & 0.0430 & 0.0560 \\
\hline Yac52265 & 0.2778 & 0.4222 & 0.7934 & 0.4440 & 0.5560 & 0.0715 \\
\hline CH14-50-6236 & 0.6611 & 0.3889 & 0.2406 & 0.3899 & 0.6101 & 0.1766 \\
\hline CH4-12-7012 & 0.1222 & 0.2222 & 1.0000 & 0.6373 & 0.3627 & 0.4831 \\
\hline Ya5NBC51 & 0.4167 & 0.4556 & 0.5259 & 0.3696 & 0.6304 & 0.0680 \\
\hline Yb7AD155 & 0.7333 & 0.4444 & 0.2922 & 0.4607 & 0.5393 & -0.0030 \\
\hline CH6-28-9163 & 0.3278 & 0.4778 & 0.4966 & 0.4240 & 0.5760 & 0.2553 \\
\hline Yb8NBC106 & 0.5889 & 0.4667 & 0.8272 & 0.3758 & 0.6242 & 0.0368 \\
\hline TARBP1R & 0.6484 & 0.4835 & 0.6513 & 0.4112 & 0.5888 & 0.0144 \\
\hline \multicolumn{4}{|l|}{ Overall } & 0.0012 & 0.0012 & 0.1224 \\
\hline \multicolumn{7}{|c|}{$\begin{array}{l}\mathrm{H}_{\mathrm{o}}=\text { Observed heterozygosity; } \mathrm{RMP}=\text { random match probability; } \mathrm{PD}=\text { power of discrimination. } \\
{ }^{1} \alpha \text {-level of } 0.05 \text { is adjusted from } 0.05 \text { to } 0.005556 \text { when corrected for multiple tests (Bonferroni's correction }\end{array}$} \\
\hline
\end{tabular}

Fig. 2. Examples of the range of the amplicon size between markers and alleles. a Null and insertion alleles of the marker LC3-2601 that have the largest difference in amplicon size. The difference between the null allele (larger amplicon) and the insertion allele (smaller amplicon) is $54 \mathrm{bp}$. b The amplicon size difference of the alleles of marker CH6-28-9163 is 1 bp. c The overall amplicon size of both alleles for the marker shown (TARBP1R) is less than $60 \mathrm{bp}$ ( 50 and $57 \mathrm{bp}$ for the insertion and null alleles, respectively), which highlights its potential use with degraded samples. 
CH4-12-7012, and CH6-28-9163) contributed more than the other loci to the overall $\mathrm{F}_{\mathrm{ST}}$. Markers that displayed lower $\mathrm{F}_{\mathrm{ST}}$ and higher heterozygosities in all three populations would tend to be more useful as individual identification markers among the three populations tested. These loci were Yac52265, Ya5NBC51, Yb7AD155, Yb8NBC106, and TARBP1R. The $\mathrm{F}_{\mathrm{ST}}$ values for these 5 loci were approximately 0.07 or less. Markers with allele frequencies that differ substantially in one or more of the populations tend to be more useful for bio-ancestral studies. For example, the locus LC3-2601, an L1 LINE, has an allele distribution approaching 50\% in the AfricanAmerican sample population but is almost monomorphic in the Hispanic and Caucasian sample populations. More than $98 \%$ of the alleles at this locus were the insertion state in Caucasians and southwestern Hispanics. Panels designed specifically around markers with similar characteristics could be very helpful in determining the ancestral lineage (or admixture) of an individual.

Similar classes of markers are the biallelic insertions and deletions (INDELs). They are appealing in that they have similar properties as INNULs, and there have been some studies describing multiplexes that could be suited for human identity testing [25, 36-42]. Indeed, there is one commercially available kit, the Investigator DIPplex Kit (Qiagen), which enables multiplex typing of $30 \mathrm{IN}$ DELs $[25,36,42]$. Another promising INDEL multiplex (with 38 markers) was described by Pereira and colleagues $[36,41]$. These INDEL multiplexes show great promise and particularly so because the alleles at a locus can be contained within short amplicons and are different but close in size. Thus, INDELs are appealing as they can be separated by capillary electrophoresis. Both of these multiplexes are informative but display relatively high $\mathrm{F}_{\mathrm{ST}}$ values and thus are not the best suited for identity testing on a global scale. The selection process to develop INDEL multiplexes with a relatively low average $\mathrm{F}_{S \mathrm{ST}}$ value will require screening a large set of potential markers [see Kidd and colleagues 43,44]. While there is a wealth of INDELs that can add to or improve upon the potential multiplexes, it may require searching for additional markers beyond known INDELs. Until now, INNULs were not amenable to PCR typing in a potentially robust manner. Because of the size difference of the null and insertion alleles, differential amplification plagued the development of assays with INNULs. This study demonstrates, for the first time, that such loci can be typed in a similar fashion to that of INDELs.

We did not develop a multiplex assay in this study. It did not make sense to devote resources to develop a mul- tiplex assay when some INNULs will be better suited for identity testing and others will be better suited for bioancestral studies. Indeed, 3 of the INNULs were not applicable to a low $\mathrm{F}_{\mathrm{ST}}$ multiplex. Before making a multiplex that could be used for human identification, it would be desirable to seek INNULs that apply well to human identity testing on a more global basis, that is, demonstrate high discrimination power and low inter-population diversity (e.g., low $\mathrm{F}_{\mathrm{ST}}$ ). The knowledge on INNUL variation in several populations is still nascent. Fortunately, the primer design described herein will facilitate screening sample populations.

\section{Conclusions}

This study demonstrates that, by utilizing the MiniPrimer strategy, INNUL markers, i.e., SINEs and LINEs, can be typed in a facile manner regardless of the size of the insertion element. The size of the amplicons for INNULs and the difference between allelic states can be reduced substantially such that these markers have utility for analyzing high- and low-quality human DNA samples in a standard PCR. Depending on the markers selected and the distribution of the alleles in global populations, INNULs can be selected for human identity testing or for bio-ancestral studies. Future work will entail selecting additional markers, developing multiplex assays, and evaluating the system(s) for forensic applications.

\section{Acknowledgements}

This work has been partially funded by the Intelligence Community Postdoctoral Research Fellowship program and was supported by the National Science Foundation Small Business Innovative Research Program (SBIR) Award No. 1113447-2011.

\section{Disclosure Statement}

B.L. LaRue, J. King, J. Ge, M. Turnbough, and B. Budowle have no conflicts of interest regarding this work.

S.K. Sinha, A.H. Montgomery, R. Thompson, and L. Klaskala are all employed by InnoGenomics, which is developing the INNUL assay as a commercially available human identification assay. 


\section{References}

1 Andersen JF, Greenhalgh MJ, Butler HR, et al: Further validation of a multiplex STR system for use in routine forensic identity testing. Forensic Sci Int 1996;78:47-64.

-2 Brinkmann B, Klintschar M, Neuhuber F, Hühne J, Rolf B: Mutation rate in human microsatellites: influence of the structure and length of the tandem repeat. Am J Hum Genet 1998;62:1408-1415.

-3 Collins PJ, Hennessy LK, Leibelt CS, Roby RK, Reeder DJ, Foxall PA: Developmental validation of a single-tube amplification of the 13 CODIS STR loci, D2S1338, D19S433, and amelogenin: the AmpFISTR ${ }^{\circledR}$ Identifil$\mathrm{er}^{\circledR}$ PCR Amplification Kit. J Forensic Sci 2004;49:1265-1277.

-4 LaFountain MJ, Schwartz MB, Svete PA, Walkinshaw MA, Buel E: TWGDAM validation of the AmpFeSTR Profiler Plus and AmpFeSTR COfiler STR multiplex systems using capillary electrophoresis. J Forensic Sci 2001;46:1191-1198.

5 Micka KA, Sprecher CJ, Lins AM, et al: Validation of multiplex polymorphic STR amplification sets developed for personal identification applications. J Forensic Sci 1996;41: 582-590.

-6 Moretti T, Baumstark AL, Defenbaugh DA, Keys KM, Smerick JB, Budowle B: Validation of short tandem repeats (STRs) for forensic usage: performance testing of fluorescent multiplex STR systems and analysis of authentic and simulated forensic samples. J Forensic Sci 2001;46:647-660.

7 Smit AF: The origin of interspersed repeats in the human genome. Curr Opin Genet Dev 1996;6:743-748.

$\$ 8$ Batzer MA, Deininger PL: Alu repeats and human genomic diversity. Nat Rev Genet 2002;3:370-379.

-9 Batzer MA, Stoneking M, Alegria-Hartman $\mathrm{M}$, et al: African origin of human-specific polymorphic Alu insertions. Proc Natl Acad Sci USA 1994;91:12288-12292.

10 Feng Q, Moran JV, Kazazian HH Jr, Boeke JD: Human L1 retrotransposon encodes a conserved endonuclease required for retrotransposition. Cell 1996;87:905-916.

11 Houck CM, Rinehart FP, Schmid CW: A ubiquitous family of repeated DNA sequences in the human genome. J Mol Biol 1979;132: 289-306.

12 Kazazian HH, Moran JV: The impact of L1 retrotransposons on the human genome. Nat Genet 1998;19:19-24.

13 Ostertag EM, Kazazian HH Jr: Biology of mammalian L1 retrotransposons. Annu Rev Genet 2001;35:501-538.

14 Ustyugova SV, Amosova AL, Lebedev YB, Sverdlov ED: Cell line fingerprinting using retroelement insertion polymorphism. Biotechniques 2005;38:561-565.
15 Novick GE, Novick CC, Yunis J, et al: Polymorphic human specific Alu insertions as markers for human identification. Electrophoresis 1995; 16:1596-1601.

16 Mamedov IZ, Shaqina IA, Kurnikova MA, et al: A new set of markers for human identification based on 32 polymorphic Alu insertions. Eur J Hum Genet 2010;18:808-814.

17 Lander ES, et al: Initial sequencing and analysis of the human genome. Nature 2001;409: 860-921.

18 Burger J, Hummel S, Hermann B, Henke W: DNA preservation: a microsatellite DNA study on ancient skeletal remains. Electrophoresis 1999;20:1722-1728.

19 Fondevila M, et al: Challenging DNA: assessment of a range of genotyping approaches for highly degraded forensic samples. Forensic Sci Int Genet Suppl Ser 2008;1:26-28.

20 Golenberg EM, Bickel A, Weihs P: Effect of highly fragmented DNA on PCR. Nucleic Acids Res 1996;24:5026-5033.

21 Hughes-Stamm SR, Ashton KJ, van Daal A: Assessment of DNA degradation and the genotyping success of highly degraded samples. Int J Legal Med 2011;125:341-348.

22 Zangenberg G, Saiki R, Reynolds R: Multiplex PCR: Optimization Guidelines. PCR Applications: Protocols for Functional Genomics. San Diego, Academic Press, 1999, pp 73-94.

23 Budowle B: SNP typing strategies. Forensic Sci Int 2004;146:S139.

-24 Syvanen AC, Sajantila A, Lukka M: Identification of individuals by analysis of biallelic DNA markers, using PCR and solid-phase minisequencing. Am J Hum Genet 1993;52: 46-59.

25 LaRue BL, Ge J, King JL, Budowle B: A validation study of the Qiagen Investigator DIPplex $^{\circledR}$ kit; an INDEL-based assay for human identification. Int J Legal Med 2012;126:533540.

26 Shriver MD, Smith MW, Lin L, Marcini A, Akey JM, Deka R, Ferrell RE: Ethnic-affiliation estimation by use of population-specific DNA markers. Am J Hum Genet 1997;60: 957-964.

27 Wang J, Song L, Grover D, Azrak S, Batzer MA, Liang P: dbRIP: a highly integrated database of retrotransposon insertion polymorphisms in humans. Hum Mutat 2006;27: 323-329.

-28 Benson DA, et al: GenBank. Nucleic Acids Res 2005;33(suppl 1):D34-D38.

29 Cheung KH, Osier MV, Kidd JR, Pakstis AJ, Miller PL, Kidd KK: ALFRED: an allele frequency database for diverse populations and DNA polymorphisms. Nucleic Acids Res 2000;28:361.
30 McGinnis S, Madden TL: BLAST: at the core of a powerful and diverse set of sequence analysis tools. Nucleic Acids Res 2004; 32(suppl 2):W20-W25.

31 Lewis PO, Zaykin D: Genetic Data Analysis: Computer Program for the Analysis of Allelic Data. Version 1.1, 2001.

32 Excoffier L, Laval G, Schneider S: Arlequin (version 3.0): an integrated software package for population genetics data analysis. Evol Bioinform Online 2005;1:47-50.

33 Weir B, Cockerham CC: Estimating F-statistics for the analysis of population structure. Evolution 1984;1358-1370.

34 Falush D, Stephens M, Pritchard JK: Inference of population structure using multilocus genotype data: linked loci and correlated allele frequencies. Genetics 2003; 164:15671587.

35 Chakraborty R: Linkage disequilibrium: concept, utility and evolutionary dynamics in the context of the human genome variation. West Lafayette, DESTOBIO 2000, 2000.

-36 Fondevila M, Phillips C, Santos C, Pereira R, Gusmão L, Carracedo A, Butler JM, Lareu MV, Vallone PM: Forensic performance of two insertion-deletion marker assays. Int J Legal Med 2012;126:725-737.

37 Li C, Zhao S, Zhang S, Li L, Liu Y, Chen J, Xue J: Genetic polymorphism of 29 highly informative InDel markers for forensic use in the Chinese Han population. Forensic Sci Int Genet 2011;5:e27-e30.

38 Weber JL, David D, Heil J, Fan Y, Zhao C, Marth G: Human diallelic insertion/deletion polymorphisms. Am J Hum Genet 2002;71: 854-862.

-39 Edelmann J, et al: Indel polymorphisms - an additional set of markers on the X-chromosome. Forensic Sci Int Genet Suppl Ser 2009; 2:510-512.

40 Pereira R, et al: Insertion/deletion polymorphisms: a multiplex assay and forensic applications. Forensic Sci Int Genet Suppl Ser 2009;2:513-515.

41 Pereira R, Phillips C, Alves C, et al: A new multiplex for human identification using insertion/deletion polymorphisms. Electrophoresis 2009;30:3682-3690.

42 Friis SL, Borsting C, Rockenbauer E, et al: Typing of 30 insertion/deletions in Danes using the first commercial indel kit - Mentype ${ }^{\circledR}$ DIPplex. Forensic Sci Int Genet 2012; 6:e72-e74.

43 Pakstis A, Speed WC, Kidd JR, Kidd KK: Candidate SNPs for a universal individual identification panel. Hum Genet 2007;121: 305-317.

44 Kidd KK, Pakstis AJ, Speed WC, et al: Developing a SNP panel for forensic identification of individuals. Forensic Sci Int 2006; 164:2032. 